\title{
Teaching Reform on The Course of Complex Analysis
}

\author{
Zhang Yong-ming \\ Mathematics teaching and research section \\ Beijing Institute of Graphic Communication, BIGC \\ Beijing ,China \\ e-mail: bjzym@bigc.edu.cn
}

\author{
Wang Dan \\ Mathematics teaching and research section \\ Beijing Institute of Graphic Communication, BIGC \\ Beijing ,China \\ e-mail: wangdan@bigc.edu.cn
}

\begin{abstract}
Mathematics experiments are applied in the course of complex analysis. By mathematical experiment, students can to chew the cud of theory, and mathematical software to calculate the results of validation, increase students ' interest in learning and using math software ability to solve mathematical problems, improve the efficiency of problem solving. According to the constructivist learning theory,in accordance with teaching, programming, manual calculation, testify to teaching the process of reform.
\end{abstract}

Keywords- Learning theories; constructivism; mathematics experiment; teaching reform

\section{INTRODUCTION}

Since the beginning of the enrollment of colleges and universities in our country in 1999, we face many new problems of higher education, during this period, the "popular education" educational thoughts and ideas become the mainstream public opinion, but really how to recognize and how to implement, it is unprecedented confusion for teachers, concerns the relationship between popular education and teaching quality. We did a serious thought, anti-wake up our own educational philosophy, combined with the development of modern educational technologies, in particular the increasing popularity of computer technology, computer technology and its applications have become readily available today use technology tools and technical resources.

Reference Literature [1] wrote to: Constructivism of learning theory is based and development up of Jean Piage tPiaget and Lev Vygotsky's thought. Constructivism believes that, learning is a process of significance construction, is a process of learners who through new old experience mutual role to formed, and rich and adjustment own of experience structure, teaching is not putting knowledge experience from external into students' mind, but to boot students from original of experience starting, growth (construction) new experiences. In today's teaching psychology, constructivism attracted researchers attention increasingly, and even (R.E.Slavin) refer to it as "a revolution in teaching of Psychology".

In accordance with the learning theory of constructivism, uniformity of traditional classroom teaching, students learning "passive" is one of the important factors affecting students ' learning effect, "spoon-feeding" teaching mode of education already cannot adapt to the new environment. So, we reduce the original classroom teaching, increased course mathematics experiment to improve students ' independent learning awareness and ability, expect students to experiment in order to hand, exert subtle influence on improving the ability of independent learning, in the lab, through repeated trial and error correction, gradual understanding and mastering (construction) new knowledge (experience).

\section{CONTENT OF TEACHING REFORM}

2.1 Reduce classroom teaching content, increase mathematics experiment

We streamline the teaching content, increasing the teaching and practice of mathematical experiments. Change the traditional way in mathematics learning to rely only on a pen and paper, tries to take advantage of modern computer technology for research on learning and traditional learning methods integration of new teaching methods.

2.2 Introduce mathematical experiment into course teaching, provide the learner with handle

On the basis of reducing contents of the classroom teaching, and the introduction of mathematics experiment into course teaching, we designed the corresponding mathematical experiment, students can experiment, continue to explore by themselves and thinking, inquiry and "autonomous" understand and grasp the knowledge learned.

We designed a integrated experiment per chapter, containing experimental content (to address of problem) and the alternate information (introduction the basic features of mathematics software function, for students to select after self-study), students can uses mathematics software completed experimental job without learning corresponding of mathematics software systemly.

At the same time, students canBe familiar with and master a practical mathematical software and some basis for future study and research.

We designed three experiments are as follows.

Experiment A Complex Integration

A.1 The experiment content

(1) Calculate the complex Integral $\int_{0}^{i} z \cos z d z$ and $\int_{-1+i}^{2+3 i} z \cos z d z$, requires simplification results.

(2) Using the Cauchy integral formula to calculate the complex integral $\oint_{|z|=5} \frac{3 z-1}{z^{2}-2 z-3} d z$, circular $|z|=5$ forward. 
(3) using higher-order derivative formula to calculate $t$ the complex integral $\oint_{|z|=5} \frac{z \sin z}{(z-i)^{2}} d z$, circular $|z|=5$ forward.

(4) Let $f(z)=u+i v, v=\mathrm{e}^{\mathrm{x}}(y \cos y+x \sin y)+x+y$, try judging $v$ is a harmonic functions and find an analytic function $\mathrm{f}(\mathrm{z})$ in this case, and fitting $\mathrm{f}(0)=1$.

A.2 Information

Some of the functions may be used:

(1) Demand the definite integral of function Integrate[function, \{integral variables, integral lower limit, integral upper limit\}];

(2) Simplify the expression of a function Simplify[expression];

(3) Function Apart[expression] -Rational expressions can be broken down into a fraction in lowest terms;

(4) Derivation function $\mathrm{D}$ [Function $\mathrm{f}$, \{self variable $x, n\}$ ] can find the function $\mathrm{f}$ on $x$ of $n$-order derivative (or partial derivative);

(5)DSolve[differential equations, the function $f(x)$, self variable $x$ ] can solve differential equations, the function $f(x)$ is the unknown function of the variable $x$, for example, DSolve $\left[\mathrm{y}^{\prime \prime}[\mathrm{x}]+2 \mathrm{y}^{\prime}[\mathrm{x}]+\mathrm{y}[\mathrm{x}]==\mathrm{x}, \quad \mathrm{y}[\mathrm{x}], \mathrm{x}\right]$, used to solve differential equations;

(6) $n$ ! represents $n$ factorial, for example $3 !=6$;

(7) $n$ !! Double factorial of $n$, for example,5!! $=5 \times 3 \times 1=15$;

(8)Factor[expression $r$ ] can give the factorization of the expression $r$, for example Factor $\left[x^{\wedge} 2+3 x+2\right]$, the expression is given the factorization results $(x+1)(x+2)$.

Experiment B series

B.1 The experiment content

(1) seeking the convergence radius of power series $\sum_{n=0}^{\infty}(1+i)^{n} z^{n}$ and $\sum_{n=2}^{\infty}\left(\frac{z}{\ln i n}\right)^{n}$.

(2) expand the function $\frac{1}{\left(1+z^{2}\right)^{2}}$ to 20-order z-series.

(3) find the 50-order Taylor expansion of function $\frac{z}{(1+z)(2+z)}$ at point $z=2$.

(4) find the Laurent series of the function $\frac{1}{z^{2}(z-i)}$ in the centre ring domain.

(5) calculate the closed integral $\int_{|z|=2} \frac{z}{1-z} e^{1 / z} d z$ using Laurent series, taking the forward path.

B.2 Information

Some of the functions may be used:

(1)Sqrt[z] can give the square root of the variable $\mathrm{z}$.

(2)Series $[f,\{x, x 0, n\}]$ can generate n-order power series at the $\mathrm{x}=\mathrm{x} 0$ of function $f$ (included in $x 0$ under the ring of Laurent series). For example, Series[1/(1+x), $\{x, 0,5\}]$ give 5order power series $1-x+x^{2}-x^{3}+x^{4}-x^{5}+o[x]^{6}$ at $x=0$ of the function $f(x)=\frac{1}{1+x}$.
(3)Normal[series s] can give the polynomials of the series $\mathrm{s}$ without remainder. For example, if $\mathrm{s}=1-x+x^{2}-x^{3}+x^{4}-x^{5}+o[x]^{6}$, then Normal[s] will get rid of more than $s+o[x]^{6}$, the polynomial results is $1-x+x^{2}-x^{3}+x^{4}-x^{5}$.

(4)SeriesCoefficient $[r, n]$ gives the coefficient of n-order term in the series $r$.

(5)Sum[f,\{i,imin,imax $\}]$, such as $\operatorname{Sum}[(-1) \wedge(n-$ 1) $/ n,\{n, 1$,Infinity $\}]$ gives the result $\ln 2$.

$\left.\operatorname{Sum}\left[(-x)^{\wedge} n\right) / 2^{\wedge} n,\{n, 1,8\}\right]$ gives the result

$-\frac{x}{2}+\frac{x^{2}}{4}-\frac{x^{3}}{8}+\frac{x^{4}}{16}-\frac{x^{5}}{32}+\frac{x^{6}}{64}-\frac{x^{7}}{128}+\frac{x^{8}}{256}$.

(6)Mathematical operations can be done between the two series.

Experiment $\mathrm{C}$ limit and derivative

C.1 The experiment content

(1) calculate $\lim _{z \rightarrow i} \frac{\operatorname{Im} z}{z}$.

(2) set $f(z)=z^{2}+i \sqrt[3]{1+z}$, calculate $f^{\prime}(z)$.

(3) set $f(z)=\left(x^{3}-3 x y^{2}\right)+i\left(3 x^{2} y-y^{3}\right)$,

determine whether it is an analytic function. If it is an analytic function, calculate its derivative.

C.2 Information

Some of the functions may be used:

(1) Limit [expression $\mathrm{f}$, trend g] can give the limit of the expression $\mathrm{f}$ when the trend $\mathrm{g}$. For example, Limit[(Tan[x]$\left.\operatorname{Sin}[\mathrm{x}]) / \mathrm{x}^{\wedge} 3, \mathrm{x}->0\right]$ can give the limit of $(\tan [x]-\sin [x]) / x^{\wedge} 3$ when $x->0$.

(2) $\mathrm{D}$ [function $\mathrm{f}$, argument $x$ ] can give the derivative of the function $\mathrm{f}$ with argument $x$. For example, $\mathrm{D}\left[\mathrm{x} \operatorname{Sin}\left[\mathrm{x}^{\wedge} 2\right], \mathrm{x}\right]$ can give the derivative of $x \operatorname{Sin}\left[x^{\wedge} 2\right]$ with $x, \mathrm{D}[\mathrm{x} \mathrm{y}, \mathrm{y}]$ can give the partial derivative of $x y$ with $y$.

(3) If [condition, execution 1, execution 2] is a conditional control function. For example, If [expression 1 $==$ expression $2, \mathrm{x}=\mathrm{a}+\mathrm{b}, \mathrm{y}=\mathrm{c}+\mathrm{d}$ ] can give $\mathrm{x}=\mathrm{a}+\mathrm{b}$ when expression $1=$ expression 2 or $y=c+d$ when expression 1 is not equal to expression 2 .

(4) Method of function definiton

For example, $\mathrm{f}\left[x_{-}\right]:=x^{\wedge} 2 \operatorname{Sin}[x]+1$ defines function $\mathrm{f}(x)=$ $x^{\wedge} 2 \sin [x]+1$, and $\mathrm{f}[1]=\sin 1+1, \mathrm{f}[2]=4 \sin 2+1$.

In order to complete the experiment, students must review the theory of knowledge and be familiar with the functions may be used of mathematical software given. In the course of the experiment, students need repeatedly to read and think, this helps students to understandd and master the knowledge, and to feel the value of theory and the powerful functions of the mathematics software.

\section{CONCLUDING REMARKS}

The mathematics experiment is designed step by step, students reduced discouraged feeling,teachers can focus on guidance.

Compared with the traditional way of teaching, we introduced mathematical experiment into the teaching process of complex analysis course, increased student's 
participation and ownership, autonomous learning of students received the attention and practice.

\section{ACKNOWLEDGMENT}

Zhang Yong-ming and Wang Dan thank the Support from PHR 201107145 and 06170112012.

\section{REFERENCES}

[1] National Commission on education technology collaborative. Introduction to theory of educational technology [m]. Beijing: higher education press, 2001,36.
[2] the Department of higher mathematics in XI ' an Jiaotong University. functions of a complex variable [m]. Beijing: higher education press, 2006.

[3] Zhang Yong-ming.Complex materials in a concluding discussion. Research on higher mathematics, Vol.10,No.4, 2007: 78-79.

[4]Zhang Yong-ming. Review on the Convergence-Divergence Tests of Alternate Series. Journal of Beijing Institute of Graphic Communication, Vol.19,No.2, 2011:70-73.

[5]Zhang Yong-ming. Review on the Convergence-Divergence Tests of Series of Infinite Constand Terms. Journal of Beijing Institute of Graphic Communication, Vol.17,No.6, 2009:67-70. 